\title{
Immunoglobulin and complement deposition in skin of rheumatoid arthritis and systemic lupus erythematosus patients
}

\author{
ARNOLD L. SCHROETER, DOYT L. CONN, AND ROBERT E. JORDON \\ From the Mayo Clinic and Mayo Foundation, Rochester, Minnesota, 55901 U.S.A.
}

\begin{abstract}
Schroeter, A. L., Conn, D. L., and Jordon, R. E. (1976). Annals of the Rheumatic Diseases, 35, 321-326. Immunoglobulin and complement deposition in skin of rheumatoid arthritis and systemic lupus erythematosus patients. Rheumatoid arthritis (RA) was differentiated from systemic lupus erythematosus (SLE) by direct immunofluorescent tenchiques on skin specimens, using monospecific antisera for IgG, IgM, C3, C1q, properdin, and fibrin. Of 30 patients with RA studied, 20 had dermal vessel deposits of immunoglobulins and complement components in unaffected skin without the characteristic dermal-epidermal junctional fluorescence of SLE. Of 24 SLE patients studied, 24 had granular deposits of immunoglobulins and complement components in unaffected skin at the dermal-epidermal junction.
\end{abstract}

Although the basic pathology of rheumatoid arthritis (RA) involves the joints, a vascular involvement of other organs has been known since the work of Bannatyne in 1898. In 1957, Bywaters described an arteritis associated with rheumatoid disease in which there was intimal proliferation of digital arteries causing small infarcts in the tissue of the periungual region of the fingers and in the digital pulp. The pathogenesis of rheumatoid vasculitis has been further delineated by the finding of immunoglobulin and complement deposition in the vasa nervorum (Conn, McDuffie, and Dyck, 1972). Consequently, the finding of immune deposits in vessels of various organs might be expected in patients with severe rheumatoid disease (Kemper, Baggenstoss, and Slocumb, 1957).

In contrast to that of RA, the clinical manifestation of systemic lupus erythematosus (SLE) commonly involves multiple organs and is a result of a possible immune complex-induced inflammation (Nydegger and others, 1974). Such immune deposits have been detected commonly at the dermal-epidermal junction of unaffected skin in SLE (Burnham, Neblett, and Fine, 1963; Gilliam and others, 1974; Tuffanelli, Kay, and Fukuyama, 1969).

This study, utilizing immunofluorescent (IF) techniques, compares the affected and the unaffected skin of RA patients with the affected and unaffected skin of SLE patients. We found that the location of the deposits of immunoglobulins and complement components in the skin in RA is different from that in active SLE.

\section{Patients}

Patients were selected who met the clinical criteria for the diagnoses of SLE and RA as designated by the American Rheumatism Association (Cohen and others, 1971; Ropes and others, 1958). All but two of the RA patients were hospitalized either for orthopaedic procedures or for treatment of severe RA. All RA patients had positive latex fixation tests for rheumatoid factor at a titre of $1: 640$ or greater.

Of the 30 patients with RA who met these criteria, 10 had uncomplicated disease with no evidence of cinical vasculitis and 20 had rheumatoid vasculitis manifested by cutaneous lesions or neuropathy (or both). All 24 patients with SLE had reactive antinuclear antibody (ANA) titres $>1: 32$ (Blondin and McDuffie, 1970).

Control studies were performed in 65 other patients. 15 normal subjects had no skin abnormalities ( 5 of these 15 were more than 50 years old) and 50 patients had skin diseases other than vasculitis.

\section{Methods}

A $4 \mathrm{~mm}$ punch was used to remove skin biopsy specimens from normal or unaffected skin of the posterior part of the calf or from skin lesions of patients with SLE or rheumatoid vasculitis. Duplicate specimens were simultaneously fixed with $10 \%$ formaldehyde, frozen immediately in liquid nitrogen, and stored at $-70^{\circ} \mathrm{C}$ for IF 
studies. Formaldehyde-fixed tissue sections were processed by haematoxylin and eosin as well as Smith elastic-Giemsa, periodic acid-Schiff, and Alcian blue stains.

Direct IF staining was performed by methods previously described (Beutner, Chorzelski, and Jordon, 1970) using monospecific conjugated antisera to IgG, IgM, IgA, C3, and fibrin. Skin biopsy specimens also were stained for $\mathrm{Clq}$, factor $\mathrm{B}$, and properdin by a modified indirect IF procedure described by Provost and Tomasi (1973) and by Rothfield and others (1972).

With the indirect method, skin sections were first treated with rabbit anti-C1q and rabbit antifactor $\mathrm{B}$, and then with conjugated goat antirabbit IgG as the second step. A similar procedure was followed when goat antiproperdin was used, except that conjugated rabbit antigoat IgG was used as the second step. Both antisera were used at a $1: 32$ dilution in the IF staining procedures. Specificity controls for these modified indirect IF staining procedures (factor B and properdin) included IF staining of tissues with the conjugated antirabbit and antigoat antisera and specific absorption of the unconjugated antisera with factor $\mathrm{B}$ and properdin (Jordon and others, 1975b).

Monospecific antiserum to human IgG was prepared, conjugated, and assayed according to the method of Triftshauser, Hayden, and Beutner (1970). Monospecific antisera to human IgA, IgM, C3, and fibrin were purchased* and tested for specificity by both immunodiffusion (Ouchterlony) and immunoelectrophoresis. Molar ratios of fluorescein to protein varied from 3.9 to 2.2. Dilution of conjugates for IF tests varied from $1: 16$ titre to $1: 40$ titre.

Antiserum to $\mathrm{Clq}$ was prepared in rabbits according to the method of Morse and Christian (1964) and was used at a 1:100 dilution in the IF testing. Antiserum to factor B was prepared in rabbits and assayed according to the method of Götze and Müller-Eberhard (1971) and used at 1:20 dilution. Antiserum to human properdin

* Hyland Division, Travenol Laboratories, Costa Mesa, CA. was prepared in a goat with properdin isolated according to the method of Pensky and others (1968), and used at a 1:10 dilution in IF testing.

\section{Results}

Immunoglobulins and complement components were found deposited in vessels in unaffected skin of patients with RA, with and without rheumatoid vasculitis (Table). Deposits of immunoglobulins (Fig. 1) coincided morphologically with deposits of the complement components in the papillary dermal and deep dermal vessels (Fig. 2). In unaffected skin the vessels in the papillary dermis showed solid and granular fluorescence. In the region of the pars papillaris, vessels appeared to be almost completely occluded by fluorescent material, with the immunoreactants deposited in the media of the vessel wall. In affected skin deep dermal vessel fluorescence was apparent and included vessels in muscular arterioles of the panniculus in addition to their presence in the papillary dermis. In both affected and unaffected skin IgM deposition occurred more often than did IgG deposition. Complement components (C3, C1q, and properdin) were found frequently. Factor $B$ deposition was not found in unaffected skin but was present in affected skin in only one of the 4 patientso As expected, fibrin deposition was present in botk unaffected and affected skin vessels.

Dermal-epidermal junctional immunofluorescence in a granular pattern was found in all patients with SLE (Fig. 3, Table). In SLE, vessel fluorescence was not found in unaffected skin and was infrequent in affected skin. Granular deposits were found in affected skin in the papillary dermal vessels in only 6 of the 22 patients. IgM was found in the media of vessels in 4 patients, compared with only one patient

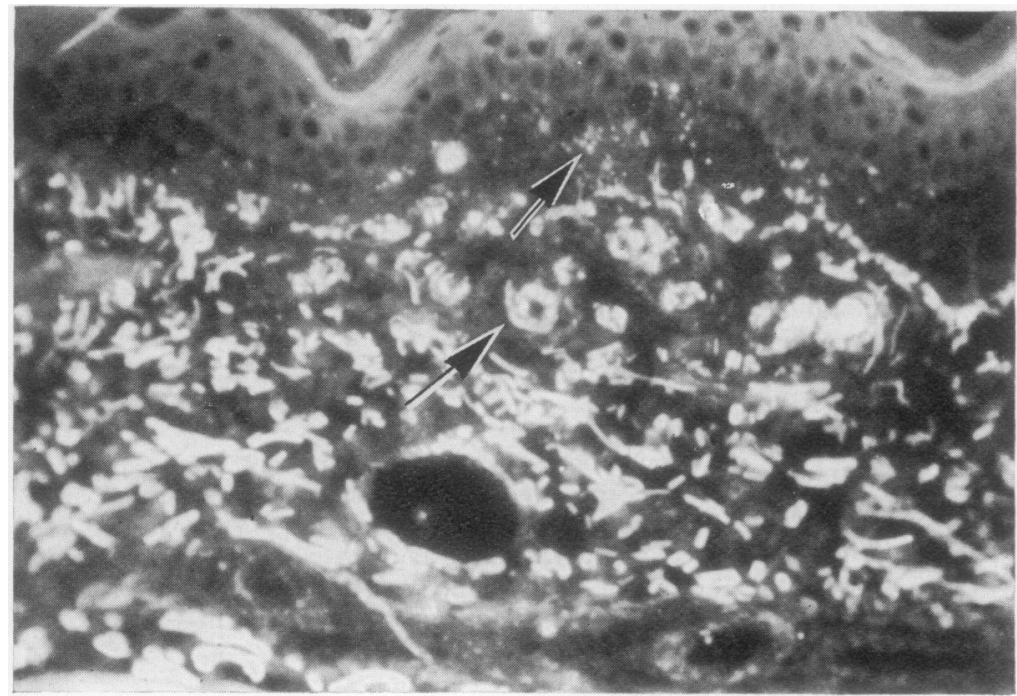

FIG. 1 IgM in unaffected skin of N rheumatoid arthritic patient. IgM deposited in papillary dermal vessels. Similar immunofluorescent patterns were seen in skin section using antisera for C3, CIq, properdin, and fibrin. $(\times 250)$ 


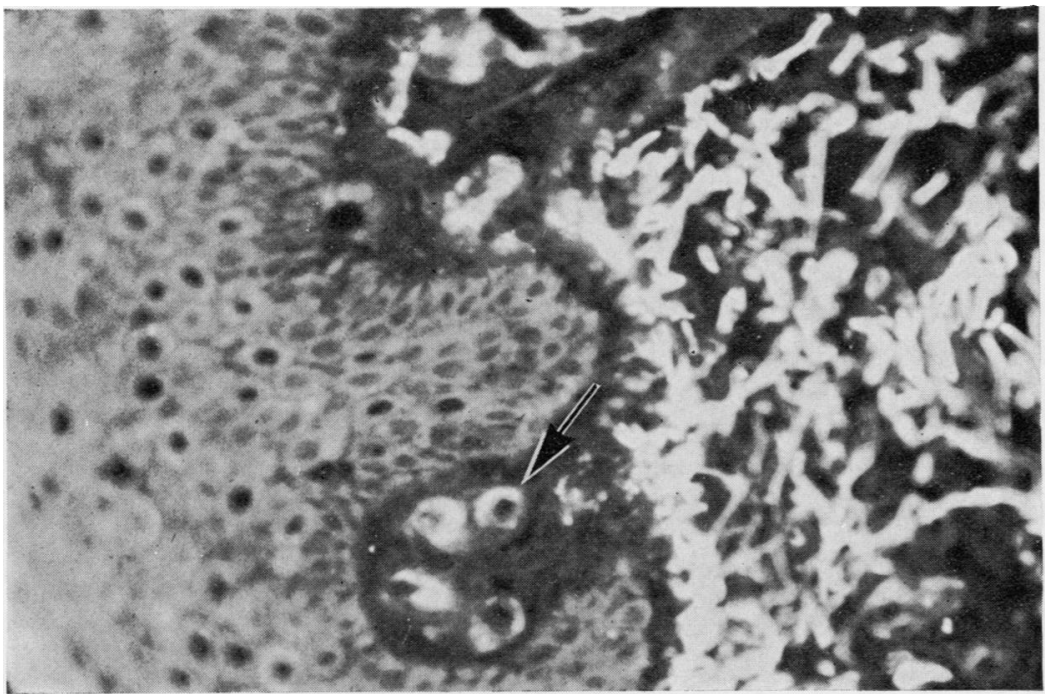

FIG. 2 C3 deposited in papillary dermal vessels in patient with rheumatoid arthritis. $(\times 250)$

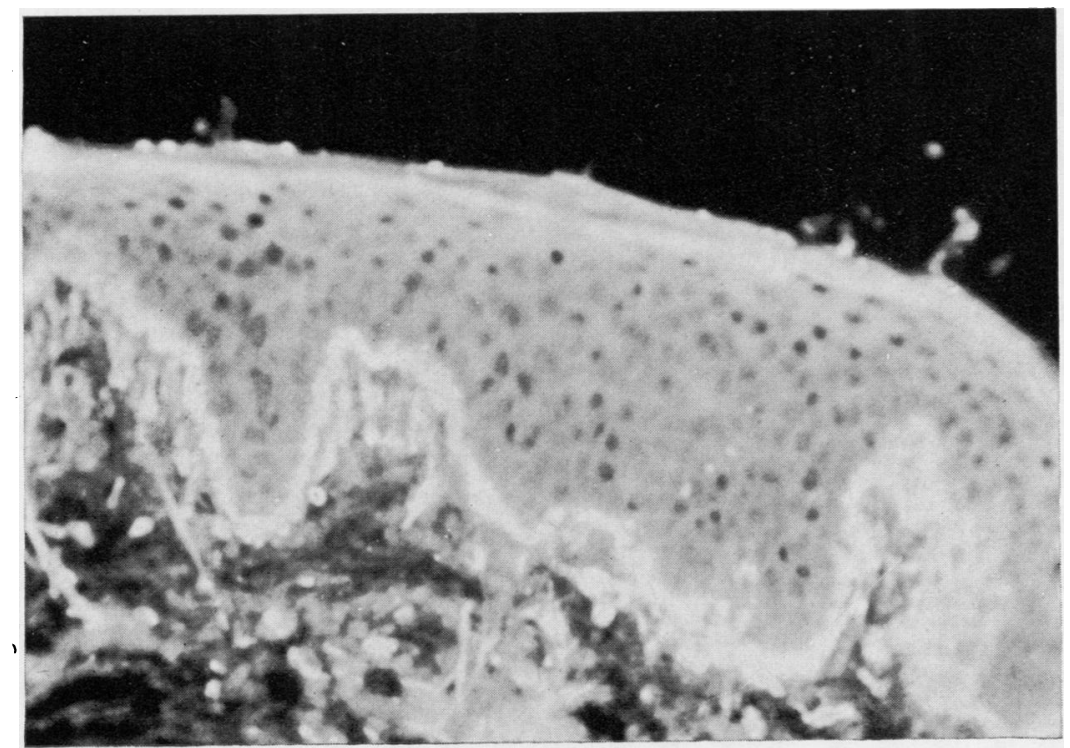

FIG. 3 Clq deposited in dermal epidermal junctional area in unaffected skin from patient with systemic lupus eryther:atosus. Similar immunofluorescent patterns were seen in serial biopsies using antisera for IgG, IgM, C3, factor $B$, properdin, and fibrin. $(\times 250)$

with IgG. Complement components (C3, Clq, properdin, and factor B) were found in some vessels in the papillary dermis of patients with affected skin. IgG was found less frequently in unaffected skin than in affected skin, whereas IgM was found with the same frequency in both and was more frequent than IgG. IgA deposits were found infrequently. Granular deposition of C1q, C3, factor B, and properdin was detected at the dermal-epidermal junction of both unaffected and affected skin as well as in the dermal vessels previously described. Complement components were found more frequently in affected skin than in unaffected skin. In both, IgG was found more commonly and fibrin less commonly in SLE than in RA.

Only one patient in the RA group had atypical, scattered, segmental, granular deposition of immunoreactants at the dermal-epidermal junction in addition to vessel fluorescence (Fig. 1). Although the patient had the typical joint deformities of RA, she also had clinical and laboratory evidence of Sjøgren's syndrome. Serological findings included a positive rheumatoid factor (titre $1: 640$ ) and ANA of $1: 256$, but a negative SLE clot test. 
Table Results of immunofluorescent studies*

\begin{tabular}{|c|c|c|c|c|}
\hline & \multicolumn{2}{|c|}{ Vessels in rheumatoid arthritis } & \multicolumn{2}{|c|}{$\begin{array}{l}\text { Dermal-epidermal junction in } \\
\text { systemic lupus erythematosus }\end{array}$} \\
\hline & $\begin{array}{l}\text { Unaffected } \\
\text { skin }\end{array}$ & $\begin{array}{l}\text { Affected } \\
\text { skin }\end{array}$ & $\begin{array}{l}\text { Unaffected } \\
\text { skin }\end{array}$ & $\begin{array}{l}\text { Affected } \\
\text { skin }\end{array}$ \\
\hline $\begin{array}{l}\text { IgG } \\
\text { IgA } \\
\text { IgM } \\
\text { C1q } \\
\text { C3 } \\
\text { Factor B } \\
\text { Properdin } \\
\text { Fibrin }\end{array}$ & $\begin{array}{r}1 / 30 \\
0 / 16 \\
20 / 30 \\
7 / 13 \\
18 / 30 \\
0 / 11 \\
7 / 16 \\
15 / 30\end{array}$ & $\begin{array}{l}1 / 4 \\
0 / 2 \\
3 / 3 \\
2 / 2 \\
3 / 4 \\
1 / 1 \\
1 / 2 \\
3 / 4\end{array}$ & $\begin{array}{r}9 / 15 \\
4 / 19 \\
15 / 15 \\
8 / 10 \\
10 / 15 \\
4 / 10 \\
3 / 10 \\
3 / 13\end{array}$ & $\begin{array}{r}20 / 22 \\
5 / 11 \\
22 / 22 \\
14 / 14 \\
20 / 22 \\
5 / 13 \\
9 / 14 \\
9 / 17\end{array}$ \\
\hline
\end{tabular}

* Shown as number positive/number tested.

There was no immunoglobulin or complement deposition in dermal vessels or at the dermalepidermal junction in the 65 controls.

The relationship between clinical features of RA and the presence of vessel fluorescence was evaluated. Of the 17 patients in the RA group who had SLE clot tests performed at least twice, 3 had positive tests without evidence of dermal-epidermal deposition of immunoglobulins or complement. Two of these 3 patients had sensory motor neuropathy and the third had vasculitis skin lesions.

\section{Discussion}

Evidence supports the occurrence of an immune complex-induced process in both RA and SLE, but the nature of the immune complexes may be different. In RA IgG complexes and diminished complement are detected in the synovial fluid of affected joints and are intimately associated with joint inflammation (Zvaifler, 1970). Patients with extra-articular manifestations of RA with skin lesions and peripheral neuropathy frequently have been found to have circulating immune complexes (Urowitz, Gordon, and Broder, 1973), diminished serum haemolytic complement (Mongan and others, 1969), IgG rheumatoid factor (Theofilopoulos and others, 1974), and the presence of a 7S IgM (Stage and Mannik, 1971). The circulating immune complexes have been characterized as being high molecular weight complexes (12-22S complexes) (Hunder and McDuffie, 1973). The presence of circulating immunoreactants in RA has been detected by a bioassay in which the immunoreactants release histamine from a guinea pig lung (Urowitz and others, 1973). These reactants have been characterized as containing IgG-IgG complexes. By use of a monoclonal IgM rheumatoid factor as a reagent, high molecular weight complexes have been detected in the sera of patients with RA with both precipitation and radioimmunoassay techniques (Luthra and McDuffie, 1974; Winchester, Kunkel, and Agnello, 1971).
Serum antinative DNA and diminished serum haemolytic complement are commonly associatediw with active SLE, particularly with lupus glomeru- $-\underset{-}{-}$ lonephritis (Schur and Sandson, 1968). Circulatingo cold insoluble complexes consisting of $\mathrm{IgG}$ and $\mathrm{Clq}-$ are found in patients who have SLE with low serum $\gg$ haemolytic complement (Stastny and Ziff, 1969). Precipitation reactions with $\mathrm{Clq}$ in gel diffusion withês gammaglobulin complexes from SLE sera can be detected. The complexes that react with $\mathrm{Clq}$ may of low molecular weight (7S) (Agnello and other; 1971). Reactivity of rheumatoid serum with C1qẩso uncommon, and cryoglobulins are uncommon in RA, perhaps indicating that different types of immune complexes circulate in the two diseases.

Immune deposits are present in unaffected skin in $\underset{\Rightarrow}{\rightarrow}$ different locations in the two diseases. In SLE there is a granular deposition of immunoglobulins and ${ }^{3}$ complement at the dermal-epidermal junction. In RA there is a solid-to-granular fluorescence in the papillary dermal vessels without dermal-epiderma junction fluorescence. The composition of theimmune deposits in both diseases is approximately the same. However, fibrin is present more commonlyo and IgG and factor B less commonly in the rheumatoid deposits, as compared with the lupus deposits.o This difference in location of immune deposits in the two diseases may be related somehow to theo difference in the size or type of circulating immune complexes.

Previous studies of affected as well as unaffected skin of patients with SLE have shown a predomi-w nance of IgG in the dermal-epidermal junctiona deposits (Gilliam and others, 1974) compared too IgM. Our studies of affected skin show the fre-D quency of IgG to be the same as that of IgM. In the unaffected skin of RA as well as of SLE patients IgMD was found more frequently than IgG. The reason? for the greater frequency of deposition of $\operatorname{IgM}$ in unaffected skin is not known at present. It is possible that IgG is present in unaffected skin but is maskedo 
by IgM (rheumatoid factor), thus making demonstration of IgG difficult. This is being investigated.

Components of both the classic and the alternate complement pathway $(\mathrm{Clq}, \mathrm{C} 3$, factor $\mathrm{B}$, and properdin) have been shown recently in the skin of patients with SLE (Jordon, Schroeter, and Winkelmann, 1975a; Provost and Tomasi, 1973; Rothfield and others, 1972). In addition, these same components, except factor B, appear in both affected and unaffected skin of RA patients. Complement deposition, however, was more frequent in affected skin.

Our finding of immunoglobulins and complement in the vessels of unaffected skin in RA does not agree with previous studies. In the studies of Muijs van de Moer and Cats (1967) and Huber and Hijmans (1971) biopsy specimens from the forearm of patients with RA who had no evidence of cutaneous vasculitis were essentially negative for immunoglobulin deposition. However, in the normal skin of the lower leg of 11 patients with RA, Larsson and Lithner (1972) detected IgG in the vessels of 6 . In our study all specimens were taken from the leg. The skin region chosen for biopsy probably has an important role in determining the frequency of positive results. The high frequency of immunoglobulin and complement in specimens taken from the leg in our study probably is related to the predilection of the clinical manifestations of vasculitis to occur in the lower extremities. Rheumatoid ulcers, purpuric lesions, and neuropathy are far more common in the leg than in the arm.

Deposition of immunoglobulins as well as complement in the dermal-epidermal junction of unaffected unexposed skin in the patient with SLE may be a useful diagnostic aid. We have shown that in RA immunoglobulins as well as complement are deposited in the papillary dermal vessels of unaffected skin. This difference in location of deposition of immune deposits may serve as a clue to the difference in the immune complexes of the two diseases. Further studies are needed to determine whether rheumatoid patients with immune deposits in papillary vessels of unaffected skin are at higher risk for developing clinical vasculitis than are patients without such deposits.

Excellent technical assistance was rendered by Mrs. Jean M. McFarland and Mrs. Jane C. Kahl. This investigation was supported in part by Research Grants AI-12049 and AM-5299 from the National Institutes of Health, Public Health Service, and by a grant from the Minnesota Chapter of the Arthritis Foundation.

\section{References}

Agnello, V., Koffler, D., Eisenberg, J. W., Winchester, R. J., AND Kunkel, H. G. (1971) J. exp. Med., 134, $228 \mathrm{~s}$ (Clq precipitins in the sera of patients with systemic lupus erythematosus and other hypocomplementemic states: characterization of high and low molecular weight types (New York Heart Association Symposium))

Bannatyne, G. A. (1898) 'Rheumatoid Arthritis: Its Pathology, Morbid Anatomy, and Treatment', 2nd ed. Wright, Bristol

Beutner, E. H., Chorzelski, T. P., AND Jordon, R. E. (1970) 'Autosensitization in Pemphigus and Bullous Pemphigoid'. Thomas, Springfield, Illinois

Blondin, C., AND McDuffie, F. C. (1970) Arthr. and Rheum., 13, 786 (Role of IgG and IgM antinuclear antibodies in formation of lupus erythematosus cells and extracellular material)

Burnham, T. K., Neblett, T. R., AND Fine, G. (1963) J. invest. Derm. 41, 451 (The application of the fluorescent antibody technic to the investigation of lupus erythematosus and various dermatoses)

Bywaters, E. G. L. (1957) Ann. rheum. Dis., 16, 84 (Peripheral vascular obstruction in rheumatoid arthritis and its relationship to other vascular lesions)

Cohen, A. J., Rfynolds, W. E., Franklin, E. C., Kulka, J. P., Ropes, M. W., Shulman, L. E., and Wallace, S. L. (1971) Bull. rheum. Dis., 21, 643 (Preliminary criteria for the classification of systemic lupus erythematosis)

ConN, D. L., MCDuffie, F. C., AND Dyck, P. J. (1972) Arthr. and Rheum., 15, 135 (Immunopathologic study of sural nerves in rheumatoid arthritis)

Gilliam, J. N., Cheatum, D. E., Hurd, E. R., Stastny, P., and Ziff, M. (1974) J."clin. Invest., 53, 1434 (Immunoglobulin in clinically uninvolved skin in systemic lupus erythematosus)

GötZe, O., AND Müller-Eberhard, H. J. (1971) J. exp. Med., 134, Suppl., 90 (The C3-activator system: an alternate pathway of complement activation)

HUBER, O., AND HIJMANS, W. (1971) 'Immunofluorescence on skin biopsies from patients with rheumatoid arthritis' in 'Rheumatoid Arthritis: Pathogenetic Mechanisms and Consequences in Therapeutics', eds. W. Müller, H.-G. Harwerth, and K. Fehr, p. 429. Academic Press, London

Hunder, G. G., AND MCDuffie, F. C. (1973) Amer. J. Med., 54, 461 (Hypocomplementemia in rheumatoid arthritis)

Jordon, R. E., Schroeter, A. L., And Winkelmann, R. K. (1975a)"Brit. J. Dermatol., 92, 263 (Dermal-epidermal deposition of complement components and properdin in systemic lupus erythematosus)

,-- Good, R. A., AND DAY, N. K. (1975b) Clin. Immun. Immunopath., 3, 307 (The complement system in bullous pemphigoid. II. Immunofluorescent evidence for both classical and alternate-pathway activation) 
Kemper, J. W., Baggenstoss, A. H., AND Slocumb, C. H. (1957) Ann. intern. Med., 46, 831 (The relationship of therapy with cortisone to the incidence of vascular lesions in rheumatoid arthritis)

LARSSON, O., AND LITHNER, F. (1972) Acta med. scand., 192, 13 (Localization of various plasma proteins in the skin in rheumatoid arthritis: an immunofluorescent microscopic study of skin biopsies)

LUTHRA, H. S., AND MCDUFFE, F. C. (1974) J. rheum., 1, Suppl. 1, 36 (Quantitation of immune complexes by radioimmunoassay)

Mongan, E. S., Cass, R. M., Jacox, R. F., and Vaughan, J. H. (1969) Amer. J. Med., 47, 23 (A study of the relation of seronegative and seropositive rheumatoid arthritis to each other and to necrotizing vasculitis)

Morse, J. H., AND Christian, C. L. (1964) J. exp. Med., 119, 195 (Immunological studies of the 11S protein component of the human complement system)

MuiJs van De MOER, W. W., AND CATS, A. (1967) Dermatologica, 134, 351 (Immunofluorescence of the skin in patients with rheumatoid arthritis: a preliminary report)

NydegGer, U. E., LAMBeRT, P. H., GeRBer, H., AND MiesCher, P. A. (1974) J. clin. Invest., 54, 297 (Circulating immune complexes in the serum in systemic lupus erythematosus and in carriers of hepatitis $B$ antigen: quantitation by binding to radiolabeled Clq)

Pensky, J., Hinz, C. F., JR., Todd, E. W., WedGWOod, R. J., Boyer, J. T., AND LePOW, I. H. (1968) J. Immunol., 100, 142 (Properties of highly purified human properdin)

Provost, T. T., AND Tomasi, T. B., JR. (1973) J. clin. Invest., 52, 1779 (Evidence for complement activation via the alternate pathway in skin diseases. I. Herpes gestationis, systemic lupus erythematosus, and bullous pemphigoid)

Ropes, M. W., Bennett, G. A., Cobb, S., JACox, R., AND Jessar, R. A. (1958) Bull. rheum. Dis., 9, 175 (1958 revision of diagnostic criteria for rheumatoid arthritis)

Rothfield, N., Ross, H. A., MiNTA, J. O., AND LePow, I. H. (1972) New Engl. J. Med., 287, 681 (Glomerular and dermal deposition of properdin in systemic lupus erythematosus)

SCHUR, P. H., AND SANDSON, J. (1968) Ibid., 278, 533 (Immunologic factors and clinical activity in systemic lupus erythematosus)

StaGe, D. E., AND MANNIK, M. (1971) Arthr. and Rheum., 14, 440 (7S $\gamma$ M-globulin in rheumatoid arthritis: evaluation of its clinical significance)

Stastny, P., AND ZiFf, M. (1969) New Engl. J. Med., 280, 1376 (Cold-insoluble complexes and complement levels in systemic lupus erythematosus)

Theofilopoulos, A. N., Burtonboy, G., LoSpalluto, J. J., AND Ziff, M. (1974) Arthr. and Rheum., 17, 272 (IgM rheumatoid factor and low molecular weight IgM: an association with vasculitis)

Triftshauser, C., Hayden, D. W., AND Beutner, E. H. (1970) Int. Arch. Allergy, 38, 315 (Procedures for the immunization of goats with human immunoglobulins and complement)

Tuffanelli, D. L., Kay, D., AND Fuxuyama, K. (1969) Arch. Derm., 99, 652 (Dermal-epidermal junction in lupus erythematosus)

Urowitz, M. B., Gordon, D. A., AND Broder, I. (1973) Arthr. and Rheum., 16, 225 (Studies into the occurrence of soluble antigen-antibody complexes in disease. V. Second assessment of correlation between the rheumatoid biologically active factor (RBAF) and the clinical features of rheumatoid arthritis)

WinCHESter, R. J., KunKel, H. G., AND AgNello, V. (1971) J. exp. Med., 134, 286 s (Occurrence of $\gamma$-globulin complexes in serum and joint fluid of rheumatoid arthritis patients: use of monoclonal rheumatoid factors as reagents for their demonstration (New York Heart Association Symposium))

ZvAIFLER, N. J. (1970) Arthr. and Rheum., 13, 895 (Further speculation on the pathogenesis of joint inflammation in rheumatoid arthritis) 